\title{
A helicity proxy from horizontal solar flow patterns
}

\author{
G. Rüdiger, Potsdam, Germany \\ Astrophysikalisches Institut Potsdam \\ A. Brandenburg, Newcastle upon Tyne, UK \\ University of Newcastle upon Tyne \\ V.V. PiPin, Irkutsk, Russia \\ Institute for Solar-Terrestrial Physics
}

Received 1999 June 25; accepted 1999 July 12

\begin{abstract}
Motivated by new observations of solar surface flow patterns of mesogranulation, theoretical computations of the horizontal divergence-vorticity correlation are presented. Because of its close relation to the helicity in rotating turbulence such observations and discussions are of particular importance for the conventional dynamo theory. For the northern hemisphere we find a small, but always negative, divergence-vorticity correlation. Both an analytical Second Order Correlation Approximation for slow rotation as well as a numerical simulation (originally done for accretion disks) for fast rotation yield very similar results.
\end{abstract}

Key words: Mesogranulation - turbulence - dynamo theory

\section{Introduction}

Modern stellar physics considers solar/stellar activity as being driven by a dynamo mechanism maintaining a largescale magnetic field. The dynamo itself is thought to result from the interaction between turbulence and rotation, the latter modifying the turbulence structure. Non-local theories for the generation of differential rotation as well as large-scale magnetic fields are described by coefficients ' $\Lambda$ ' and ' $\alpha$ ' that capture the influence of the basic rotation on the turbulence.

In the present paper we ask the question whether the rotational influence on the turbulence can be observed at the solar surface. Granulation and mesogranulation are the main candidates due to their nonmagnetic character. Our particular question is whether the horizontal motions alone suffice to estimate the rotational influence.

The helicity $\left\langle\boldsymbol{u}^{\prime} \cdot \operatorname{curl} \boldsymbol{u}^{\prime}\right\rangle$ is a key ingredient in conventional dynamo theory (Krause \& Rädler 1980). Here we consider that part of the helicity which results from the vertical components of velocity and vorticity,

$$
\mathcal{H}=\left\langle w\left(\frac{\partial v}{\partial x}-\frac{\partial u}{\partial y}\right)\right\rangle
$$

where $(u, v, w)=\boldsymbol{u}^{\prime}$ denote the deviations from the mean flow in a Cartesian coordinate system $(x, y, z)$. The angular brackets denote ensemble averages, which are in practice approximated by finite spatial and temporal averages. We adopt a right-handed coordinate system (e.g., if $x$ points east, $y$ north, then $z$ points radially outwards.) It is well known (e.g. Krause \& Rädler 1980) that in a stratified convection zone rising material expands and rotates because of the action of the Coriolis force. On the northern hemisphere the results are lefthanded helical motions, i.e. $\mathcal{H}<0$. Expansion results in clockwise rotation and vice versa. Instead of (1) we consider now the divergence-vorticity correlation

$$
\mathcal{C}=\left\langle\left(\frac{\partial u}{\partial x}+\frac{\partial v}{\partial y}\right)\left(\frac{\partial v}{\partial x}-\frac{\partial u}{\partial y}\right)\right\rangle
$$

(cf. Wang et al. 1995). In the following we refer to this quantity as a helicity proxy. We adopt the anelastic approximation, $\operatorname{div} \rho \boldsymbol{u}=0$, and neglect fluctuations in the density, i.e. $\rho=\rho(z)$, so $\operatorname{div} \rho \boldsymbol{u}^{\prime}=0$. Thus, we have

$$
\frac{\partial u}{\partial x}+\frac{\partial v}{\partial y}+\frac{\partial w}{\partial z}+w \frac{\partial \ln \rho}{\partial z}=\frac{\partial u}{\partial x}+\frac{\partial v}{\partial y}+w \frac{\partial \ln |\rho w|}{\partial z}=0
$$


We now define a scale height for the vertical momentum fluctuations,

$$
H_{\mathrm{m}}=-\left\langle\frac{\partial \ln |\rho w|}{\partial z}\right\rangle^{-1}
$$

so the horizontal velocity divergence is then approximately $w / H_{\mathrm{m}}$, and therefore

$$
\mathcal{C} \approx\left\langle\frac{w}{H_{\mathrm{m}}}\left(\frac{\partial v}{\partial x}-\frac{\partial u}{\partial y}\right)\right\rangle=\frac{\mathcal{H}}{H_{\mathrm{m}}} .
$$

We assume $H_{\mathrm{m}}>0$, i.e. the vertical momentum fluctuations decrease with height. This is indeed a natural property of many granulation models (cf. Simon \& Weiss 1997). Note that even if $|w|$ increases mildly with height $H_{\mathrm{m}}$ can still be positive. Close to the bottom of the cells, however, $H_{\mathrm{m}}$ becomes negative. The simulations presented in Sect. 3 indicate that this approximation is relatively accurate. Thus, from

$$
\frac{\partial u}{\partial x}+\frac{\partial v}{\partial y} \approx \frac{w}{H_{\mathrm{m}}}
$$

we see that in the top realm of the cells a positive horizontal divergence corresponds to an updraft $(w>0)$ and a negative horizontal divergence corresponds to a downdraft $(w<0)$, which is in agreement with our expectation.

Given that $\mathcal{C}$ is closely related to $\mathcal{H}$, it should be possible to estimate $\mathcal{H}$ from observations of horizontal flow components alone. In the Second Order Correlation Approximation (SOCA) the sign of the helicity is related to the sign of the $\alpha$-effect. For isotropic $\alpha$-effect negative helicity leads to positive $\alpha$ and vice versa - both for low and high conductivity (Krause \& Rädler 1980),

$$
\alpha \simeq-\tau_{\text {corr }} \mathcal{H}
$$

with $\tau_{\text {corr }}$ as the correlation time in the turbulent flow field. Positive values of $\alpha$ are thus expected for the northern solar hemisphere. ${ }^{1}$ However, with a positive $\alpha$-effect and a rotation law taken from helioseismology inversions, the solar dynamo produces the wrong butterfly diagram (see Parker 1987). It would, therefore, be useful, to observe the helicity in the solar atmosphere in order to put constraints on dynamo theory.

Like the helicity $\mathcal{H}$, so is also $\mathcal{C}$ a pseudoscalar and its sign depends on the coordinate system. The only pseudoscalar, that can be constructed in anisotropic turbulence with the anisotropy direction $\boldsymbol{g}$, is the scalar product $\boldsymbol{g} \cdot \boldsymbol{\Omega}$, where $\boldsymbol{\Omega}$ is the angular velocity vector. Hence, we expect a nonvanishing helicity proxy $\mathcal{C}$ only for rotating turbulence. The influence of the basic rotation is often represented by the Coriolis number (or inverse Rossby number)

$$
\Omega^{*}=2 \tau_{\text {corr }} \Omega
$$

i.e. the ratio between correlation time $\tau_{\text {corr }}$ and the rotation period.

As the typical mesogranulation pattern only lives for a few hours $\Omega^{*}$ is estimated to be of order of $10^{-1}$. Hence, the correlation effect $\mathcal{C}$ can only be very small and one will need a sophisticated observational strategy to measure it. In Brandt et al. (1988) and in Simon et al. (1994) first results of an overall inspection of horizontal flow patterns on mesoscales are presented. The maximum velocities are $\sim 750 \mathrm{~m} / \mathrm{s}$, maximum vertical divergence is $\sim 4 \cdot 10^{-4} \mathrm{~s}^{-1}$ and the maximum vertical vorticity is $\sim 2 \cdot 10^{-4} \mathrm{~s}^{-1}$ (see also Simon et al. 1988). Brandt et al. (1988) even report a vorticity of an order of magnitude larger than those values. There are indications for a negative divergence-vorticity correlation $\mathcal{C}<0$ (Wang et al. 1995) but a more detailed analysis must bring the final finding about the rather small effect. The best chance of detection may be expected if the averages are taken over large enough scales.

\section{Influence of the basic rotation}

A vertical unit vector $\stackrel{\circ}{g}$ may be introduced. The desired expression (2) can generally be written as the $z$-component of the axial vector

$$
\mathcal{C}=\left\langle\operatorname{div} \tilde{\boldsymbol{u}} \operatorname{curl} \boldsymbol{u}^{\prime}\right\rangle
$$

with $\tilde{\boldsymbol{u}}=\boldsymbol{u}^{\prime}-\left(\stackrel{\circ}{\boldsymbol{g}} \cdot \boldsymbol{u}^{\prime}\right) \stackrel{\circ}{\boldsymbol{g}}$ as the horizontal component of the random flow field. Angular brackets denote ensemble averages.

\footnotetext{
${ }^{1}$ The situation is more complicated if the tensor character of the $\alpha$-effect is taken into account, but also in this case the important azimuthal component of the $\alpha$-tensor, $\alpha_{\phi \phi}$, is positive in the northern hemisphere (Kitchatinov \& Rüdiger 1993).
} 
It makes sense to transform the latter to the random momentum field with $\boldsymbol{m}^{\prime}=\rho \boldsymbol{u}^{\prime}$, i.e.

$$
\rho \operatorname{curl} \boldsymbol{u}^{\prime}=\operatorname{curl} \boldsymbol{m}^{\prime}+\boldsymbol{m}^{\prime} \times \boldsymbol{G}, \quad \rho \operatorname{div} \boldsymbol{u}^{\prime}=-\boldsymbol{G} \cdot \boldsymbol{m}^{\prime}
$$

with $\boldsymbol{G}=\nabla \ln \rho$ and with the mass conservation law in an anelastic medium, $\operatorname{div} \boldsymbol{m}^{\prime}=0$. It follows $\rho \stackrel{\circ}{g} \cdot$ curl $\boldsymbol{u}^{\prime}=$ $\stackrel{\circ}{\boldsymbol{g}} \cdot \operatorname{rot} \boldsymbol{m}^{\prime}$ and $\rho \operatorname{div} \tilde{\boldsymbol{u}}=\operatorname{div} \tilde{\boldsymbol{m}}$ so that

$$
\mathcal{C}=-\frac{1}{\rho^{2}}\left\langle(\stackrel{\circ}{\boldsymbol{g}} \cdot \nabla)\left(\stackrel{\circ}{\boldsymbol{g}} \cdot \boldsymbol{m}^{\prime}\right)\left(\operatorname{curl} \boldsymbol{m}^{\prime}+\boldsymbol{m}^{\prime} \times \boldsymbol{G}\right)\right\rangle
$$

where the nonfluctuating density profile $\rho=\rho(z)$ has been pulled out of the average. With the Fourier transform

$$
\boldsymbol{m}^{\prime}(\boldsymbol{x}, t)=\int \hat{\boldsymbol{m}}(\boldsymbol{k}, \omega) e^{\mathrm{i}(\boldsymbol{k} \cdot \boldsymbol{x}-\omega t)} d \boldsymbol{k} d \omega
$$

we obtain

$$
\begin{aligned}
\rho^{2} \mathcal{C}_{i}= & \epsilon_{i j n} \stackrel{\circ}{g}_{f} \stackrel{\circ}{g}_{m} \\
& \int k_{j}^{\prime} k_{m}\left\langle\hat{m}_{f}(\boldsymbol{k}, \omega) \hat{m}_{n}\left(\boldsymbol{k}^{\prime}, \omega^{\prime}\right)\right\rangle e^{\mathrm{i}\left(\boldsymbol{k}+\boldsymbol{k}^{\prime}\right) \cdot \boldsymbol{x}-\left(\omega+\omega^{\prime}\right) t} d \boldsymbol{k} d \boldsymbol{k}^{\prime} d \omega d \omega^{\prime}
\end{aligned}
$$

for the components of $\mathcal{C}$. Here $\epsilon_{i j n}$ is the totally antisymmetric tensor.

In general, the axial vector (9) can only be expressed by another axial vector. To this end the axial vector of the angular velocity can be used, so that we write

$$
\mathcal{C}_{i}=C_{i j} \Omega_{j}
$$

with the coefficient tensor

$$
C_{i j}=c_{0} \stackrel{\circ}{g}_{i} \stackrel{\circ}{g}_{j}+c_{1} \delta_{i j}+c_{2} \epsilon_{i j p} \Omega_{p}+c_{3} \Omega_{i} \Omega_{j}+c_{4}(\stackrel{\circ}{g} \cdot \Omega)^{2} \delta_{i j}
$$

- up to the second order in $\Omega$. Note that the term with $c_{2}$ does not contribute to (14). For small (8) one can use the slow-rotation approximation which only involves the coefficients $c_{0}$ and $c_{1}$. Then the latitudinal component of the correlation becomes

$$
\mathcal{C}_{\theta}=-c_{1} \sin \theta \Omega
$$

This correlation, however, must vanish at the equator for equatorsymmetric flows. If we thus assume the mesogranulation pattern as equatorsymmetric, $c_{0}$ proves to be the only interesting coefficient, hence for $\mathcal{C} \equiv \stackrel{\circ}{g}_{i} \mathcal{C}_{i}$

$$
\mathcal{C}=c_{0} \cos \theta \Omega
$$

results. To first order in $\Omega$ the correlation vector $\mathcal{C}$ has only a radial (here: vertical) component, i.e. $\mathcal{C}$.

It remains to compute the tensor (15). In a stratified turbulent medium the spectral tensor in (13) is

$$
\begin{aligned}
\hat{M}_{i j}= & \left\langle\hat{m}_{i}(\boldsymbol{k}, \omega) \hat{m}_{j}\left(\boldsymbol{k}^{\prime}, \omega^{\prime}\right)\right\rangle=\frac{E(K, \omega, \kappa)}{16 \pi k^{2}} \\
& \left(\delta_{i j}-\frac{K_{i} K_{j}}{K^{2}}+\frac{\kappa_{i} K_{j}-\kappa_{j} K_{i}}{2 K^{2}}+\frac{K^{2} \kappa_{i} \kappa_{j}-\kappa^{2} K_{i} K_{j}}{4 K^{4}}\right) \delta\left(\omega+\omega^{\prime}\right),
\end{aligned}
$$

where $\boldsymbol{K}$ and $\boldsymbol{\kappa}$ are the wave vectors for large as well as small scales (Kitchatinov \& Rüdiger 1993). It is simply $\boldsymbol{K}=\left(\boldsymbol{k}-\boldsymbol{k}^{\prime}\right) / 2$ and $\boldsymbol{\kappa}=\boldsymbol{k}+\boldsymbol{k}^{\prime}$.

Insertion of (18) into (13) does not provide any nonvanishing values. Only the influence of the basic rotation onto the turbulence produces a finite effect. In Kitchatinov \& Rüdiger (1993) the rotational influence is computed in full detail. The results must be introduced into (13), which after massive manipulations leads to

$$
\begin{gathered}
\mathcal{C}_{i j}=-\frac{3}{70 \rho^{2}} \frac{\partial^{2}}{\partial x_{i} \partial x_{j}} \int \nu k^{2} E(k, \omega, \boldsymbol{r}) \frac{\nu^{2} k^{4}+7 \omega^{2}}{\left(\omega^{2}+\nu^{2} k^{4}\right)^{2}} d k d \omega- \\
-\frac{G_{i}}{35 \rho^{2}} \frac{\partial}{\partial x_{j}} \int E(k, \omega, \boldsymbol{r}) \frac{\nu k^{2}}{\omega^{2}+\nu^{2} k^{4}} d k d \omega
\end{gathered}
$$

for uniform stratification $\boldsymbol{G}$. The result written here is of first order in the rotation rate and for uniform density stratification $\boldsymbol{G}$. After adopting the mixing length approximation the result is

$$
\mathcal{C}_{i j}=-\left[\frac{3}{70 \rho^{2}} \frac{\partial^{2}}{\partial x_{i} \partial x_{j}}\left(\rho^{2}\left\langle u^{\prime 2}\right\rangle \tau_{\text {corr }}\right)+\frac{G_{i}}{35 \rho^{2}} \frac{\partial}{\partial x_{j}}\left(\rho^{2}\left\langle u^{\prime 2}\right\rangle \tau_{\text {corr }}\right)\right] .
$$


Note after comparison that indeed no Kronecker tensor $\delta_{i j}$ appears in the expression (15). When the inhomogeneity of the turbulence intensity can be neglected compared with that of the density stratification,

$$
\mathcal{C}=-\frac{8}{35}(\boldsymbol{G} \cdot \stackrel{\circ}{\boldsymbol{g}})(\boldsymbol{G} \cdot \boldsymbol{\Omega})\left\langle u^{2}\right\rangle \tau_{\text {corr }}
$$

is obtained for the vertical component of the correlation vector (14). The resulting helicity proxy,

$$
\mathcal{C}=-\frac{8}{35} \frac{\alpha_{\text {MLT }}^{2}}{\gamma^{2}} \frac{\Omega}{\tau_{\text {corr }}} \cos \theta
$$

proves to be negative on the northern hemisphere - as expected from (5). Here, $\alpha_{\text {MLT }}$ is the standard mixing length parameter and $\gamma$ is the ratio of specific heats and we have used typical relations such as $\tau_{\text {corr }} u^{\prime} \approx l_{\text {corr }}$. The resulting helicity proxy for mesogranulation is only of order $10^{-10} \mathrm{~s}^{-2}$. Hence, it must be a very delicate observational problem to find out the rotational influence with the turbulent pattern at the solar surface as given in (22).

\section{Simulations}

In order to test the feasibility of estimating $\mathcal{H}$ from horizontal velocity measurements we now analyse data from numerical simulations of stratified shear flow turbulence. Those data are particularly interesting, because the flow supports large scale dynamo action. All details about this simulation can be found in Brandenburg et al. (1995). For the present purpose it suffices to say that the simulation covers part of an accretion disc, where the density varies by a factor of thirty in the $z$-direction between the midplane and the top and bottom surfaces. The inverse Rossby number, $\Omega^{*}$, is around 20 and the Reynolds number with respect to the turbulence is around 100 , and with respect to the shear flow around 750 . The relative helicity of the flow, i.e. the helicity normalized with respect to the root-mean-square values of vorticity and velocity is less than $5 \%$. We mention this, because it is important to realise that even though the system is rapidly rotating and stratified, the helicity is well below the maximum possible value. This is partly because significant cancellation is taking place from flow regions where the helicity may locally be large, but of either sign.

We now proceed by estimating the helicity from horizontal velocity measurements. In Figure 1 we show a correlation plot between horizontal divergence and vorticity for a particular location in the box. The value of the helicity proxy, normalized by the mean square of the horizontal velocity divergence,

$$
\mathcal{C}_{\text {slope }}=\frac{\left\langle\left(\frac{\partial u}{\partial x}+\frac{\partial v}{\partial y}\right)\left(\frac{\partial v}{\partial x}-\frac{\partial u}{\partial y}\right)\right\rangle}{\left\langle\left(\frac{\partial u}{\partial x}+\frac{\partial v}{\partial y}\right)^{2}\right\rangle},
$$

is just the slope in the correlation plot (Figure 1). The plus signs in Figure 3. give the dependence of $\mathcal{C}_{\text {slope }}$ on $z / H$, where $H$ is the gaussian density scale height. The asterisks give the relative helicity proxy,

$$
\mathcal{C}_{\text {rel }}=\frac{\left\langle\left(\frac{\partial u}{\partial x}+\frac{\partial v}{\partial y}\right)\left(\frac{\partial v}{\partial x}-\frac{\partial u}{\partial y}\right)\right\rangle}{\left\langle\left(\frac{\partial u}{\partial x}+\frac{\partial v}{\partial y}\right)^{2}\right\rangle^{1 / 2}\left\langle\left(\frac{\partial v}{\partial x}-\frac{\partial u}{\partial y}\right)^{2}\right\rangle^{1 / 2}},
$$

which is just the correlation index. The significance of the correlation is difficult to assess. The points in the correlation plot are not all independent, because nearby points are coupled through viscosity. However, if we assume that every 4th meshpoint in each of the two horizontal directions is independent we still have about $n=2000 / 16=125$ independent points, so the 95 -percent confidence level is $2 / \sqrt{n} \approx 0.18$. The magnitude of the correlation index is at many locations just above the 95-percent confidence level, but often even below.

We find that $\mathcal{C}$ is positive in the lower part of the midplane and negative above. This is consistent with the expected, and directly measured, sign of the helicity. However, the scatter is very large. Thus, in order to obtain a reliable result from observations of solar mesogranulation, it is important to produce good averages using data at many different times to eliminate the naturally occurring fluctuations of the turbulence.

We may conclude by saying that the rotational influence on the turbulence may be observable, but it is vital to generate good averages using independent data obtained at different times to see this relatively small effect. If observations really confirm that the helicity is negative in the northern hemisphere, then Eq. (7) would suggest that the $\alpha$-effect is positive there. This leads directly to the 'dynamo dilemma' (Parker 1987). If indeed both the helicity concept and the model calculations are correct, then we have to question the relation (7) more seriously.

Acknowledgements. We are very grateful for discussions with P. N. Brandt (Freiburg) who drew our attention to the observational findings considered in the present paper. V. V. Pipin acknowledges support through the visitor program of the Astrophysical Institute Potsdam. 


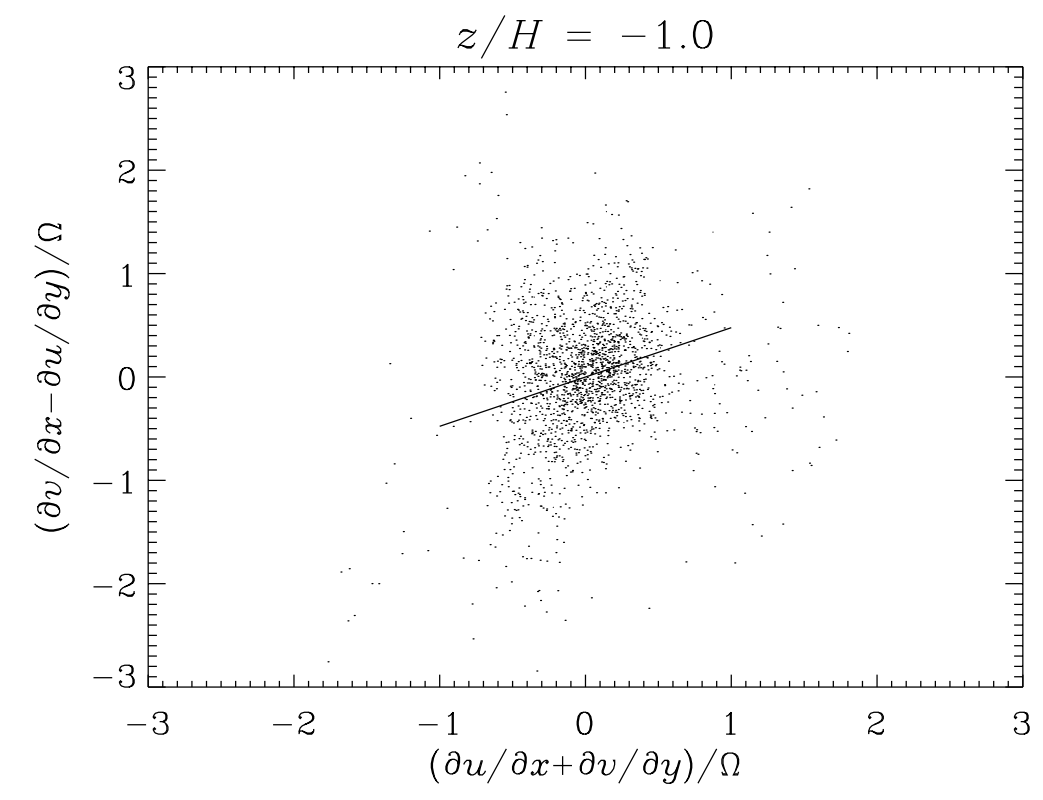

Fig. 1: Scatter plot between horizontal divergence and horizontal vorticity (in units of the angular velocity $\Omega$ ) in a horizontal plane for $z / H=-1.0$. The positive correlation indicates positive helicity for negative values of $z$. Note also that the scatter is relatively large.

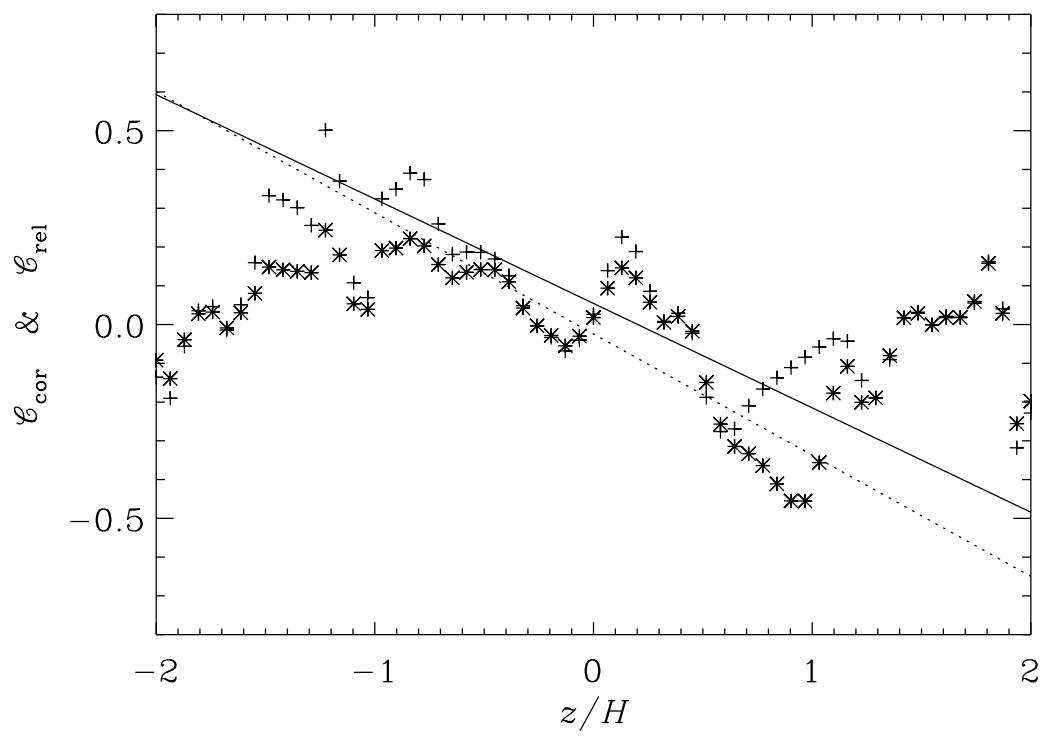

Fig. 2: $\mathcal{C}_{\text {slope }}$ (plus signs) and $\mathcal{C}_{\text {rel }}$ (asterisks) as a function of $z / H$. The solid line gives a least-square fit through the plus signs. 


\section{References}

Brandenburg, A., Nordlund, Å., Stein, R. F., Torkelsson, U.: 1995, Astrophys. J. 446, 741

Brandt, P.N., Scharmer, G.B., Ferguson, S.H., Shine, R.A., Tarbell, T.D., Title, A.M.: 1988, Nature 335, 238

Kitchatinov, L.L., Rüdiger, G.: 1993, Astron. Astrophys. 276, 96

Krause, F., Rädler, K.-H.: 1980, Mean-Field Magnetohydrodynamics and Dynamo Theory, Akademieverlag, Berlin

Parker, E.N.: 1987, Sol. Phys. 110, 11

Simon, G.W., Title, A.M., Topka, K.P., Tarbell, T.D., Shine, R.A., Ferguson, S.H., Zirin, H.: 1988, Astrophys. J. 327, 964

Simon, G.W., Brandt, P.N., November, L.J., Scharmer, G.B., Shine, R.A.: 1994, in R.J. Rutten and C.J. Schrijver (eds.) Solar Surface Magnetism, Kluwer Academic Publishers, Dordrecht, p. 261

Simon, G.W., Weiss, N.O.: 1997, Astrophys. J. 489, 960

Wang, Y., Noyes, R.W., Tarbell, Th. D., Title, A.M.: 1995, Astrophys. J. 447, 419

Addresses of the authors:

Günther Rüdiger, Astrophysikalisches Institut Potsdam, An der Sternwarte 16, D-14482 Potsdam, Germany, e-mail: GRuediger@aip.de

Axel Brandenburg, Department of Mathematics, University of Newcastle upon Tyne, NE1 7RU, England, UK, e-mail: Axel.Brandenburg@newcastle.ac.uk

V.V. Pipin, Institute for Solar-Terrestrial Physics, P.O. Box 4026, Irkutsk, 664033, Russia, e-mail: pip@iszf.irk.ri 